\title{
JOURNAL

\section{Biomechanical strategies for successful obstacle crossing with the trailing limb in older adults with medial compartment knee osteoarthritis}

\author{
Hao-Ling Chen ${ }^{\mathrm{a}, \mathrm{b}, 1}$, Tung-Wu Lu ${ }^{\mathrm{a}, *, 1}$, Ting-Ming Wang ${ }^{\mathrm{a}, \mathrm{c}}$, Shier-Chieg Huang ${ }^{\mathrm{d}}$ \\ ${ }^{a}$ Institute of Biomedical Engineering, National Taiwan University, Taiwan, ROC \\ ${ }^{\mathrm{b}}$ School of Occupational Therapy, National Taiwan University, Taiwan, ROC \\ ${ }^{\mathrm{c}}$ Department of Orthopaedic Surgery, National Taiwan University Hospital, Taiwan, ROC \\ ${ }^{\mathrm{d}}$ Department of Orthopaedic Surgery, National Taiwan University Hospital Yun-Lin Branch, Taiwan, ROC
}

Accepted 23 November 2007

\begin{abstract}
To investigate the biomechanical strategy adopted by older adults with medial compartment knee osteoarthritis (OA) for successful obstacle crossing with the trailing limb, and to discuss its implications for fall-prevention, 15 older adults with bilateral medial compartment knee OA and 15 healthy controls were recruited to walk and cross obstacles of heights of $10 \%, 20 \%$, and $30 \%$ of their leg lengths. Kinematic and kinetic data were obtained using a three-dimensional (3D) motion analysis system and forceplates. The OA group had higher trailing toe clearance than the controls. When the trailing toe was above the obstacle, the OA group showed greater swing hip abduction, yet smaller stance hip adduction, knee flexion, and ankle eversion. They showed greater pelvic anterior tilt and toe-out angle. They also exhibited greater peak knee abductor moments during early stance and at the instant when the swing toe was above the obstacle, while a greater peak hip abductor moment was found during late stance. Smaller knee extensor, yet greater hip extensor moments, were found in the OA group throughout the stance phase. In order to achieve higher toe clearance with knee OA, particular joint kinematic and kinetic strategies have been adopted by the OA group. Weakness in the hip abductors and extensors in individuals with OA may be risk factors for tripping owing to the greater demands on these muscle groups during obstacle crossing by these individuals.
\end{abstract}

(C) 2007 Elsevier Ltd. All rights reserved.

Keywords: Knee osteoarthritis; Gait; Obstacle crossing; Joint kinematics; Joint kinetics

\section{Introduction}

Falls are a leading cause of death in the elderly in the USA (Hoyert et al., 2001); one of the most frequent causes of which is tripping during obstacle negotiation (Blake et al., 1988; Campbell et al., 1990; Overstall et al., 1977). During obstacle crossing, the leading limb must first cross the obstacle successfully, followed by the trailing limb. When the trailing limb is crossing, neither the swing limb nor the obstacle is within the subject's visual field. Without visual feedback about the relative positions of the swing

\footnotetext{
${ }^{*}$ Corresponding author. Tel./fax: + 886233653335.

E-mail address: twlu@ntu.edu.tw (T.-W. Lu).

${ }^{1}$ These authors contributed equally to this work.
}

limb and obstacle, control of the motion may become even more difficult if the neuromusculoskeletal system is affected either by injury or disease. In fact, knee osteoarthritis (OA), a common joint disorder, has been identified as a factor predisposing the elderly to falls (Leveille et al., 2002; Robbins et al., 1989). Older people with knee OA may have an increased risk of falling during obstacle crossing with the trailing limb as the number of risk factors increases (Tinetti et al., 1988).

Obstacle crossing during walking requires precise control of the swing foot while maintaining body balance through highly coordinated joint movements of both limbs. Since before crossing the trailing foot is placed on the floor closer to the obstacles than the leading foot (Chou and Draganich, 1997), the trailing limb has a shorter time and 
distance for the preparation of a safe crossing with sufficient clearance. Previous studies showed that toe clearances of the trailing limb were smaller than those of the leading limb in the healthy elderly ( $\mathrm{Lu}$ et al., 2006; McFadyen and Prince, 2002), suggesting that an increased risk of tripping owing to toe-obstacle contact may occur when the trailing limb is crossing. Apart from the joint kinematics of the swing limb, well-controlled joint kinematics and kinetics of the stance limb are also important for maintaining sufficient foot clearance during obstacle crossing. Knowledge about the joint mechanics of the trailing stance limb during leading limb crossing is well established (Chou and Draganich, 1997; Draganich and Kuo, 2004; McFadyen and Prince, 2002). However, studies on both the trailing swing and leading stance limbs during trailing limb crossing are limited (Chen and Lu, 2006).

The performance of patients with knee OA during obstacle crossing has been studied only fairly recently ( $\mathrm{Lu}$ et al., 2007; Pandya et al., 2005, 2007). Under test conditions, these patients had an increased chance of tripping over obstacles, compared to their age-matched controls (Pandya et al., 2005). Obstacle avoidance success rates increased with pain relief, but remained less than those of the healthy subjects (Pandya et al., 2007). Lu et al. (2007) found that patients with bilateral medial compartment knee OA adopted particular kinematic and kinetic strategies to compensate for the compromised function of the diseased knees and their surrounding muscles during obstacle crossing with the leading limb. However, it remains unknown whether different strategies would be used when crossing with the trailing limb. To address this issue, data collected from the same subject groups during the previous study by $\mathrm{Lu}$ et al. (2007) were used in the current study to investigate the biomechanical strategies adopted by older adults with medial compartment knee OA for successful obstacle crossing of the trailing limb. Biomechanical differences between the crossing of the leading and the trailing limbs are also discussed.

\section{Materials and methods}

\subsection{Subjects}

Fifteen patients with knee OA were recruited by an orthopedic surgeon (T.M.W.). All subjects met the following inclusion criteria: (a) age 65 years or older, (b) bilateral medial compartment knee OA, (c) both knees were of grade 2 or higher according to the standards of Kellgren/Lawrence (K/L) (Kellgren and Lawrence, 1957), (d) ability to cross the obstacle without assistance, and (e) no excessive pain affecting their gait, with a visual analog scale (VAS) score less than $6(0=$ no pain, $10=$ worst imaginable pain; Dalton et al., 1998). Subjects were not included if they had other neuromusculoskeletal diseases which may affect gait and/or cognitive function, such as rheumatoid arthritis, neuropathic arthropathy, stroke, and fracture. If the subjects had had intra-articular corticosteroid injection in the previous 2 months or had planned for a total knee replacement in the following year, they were also excluded. Fifteen healthy individuals without a history of $\mathrm{OA}$ were recruited by advertisement to serve as age-matched and sex-matched control subjects. All subjects in the study had normal or corrected-to-normal vision and were free of neuromusculoskeletal pathology. Ethics approval was granted by the Institutional Human Research Ethics Committee.

\subsection{Gait analysis}

In a gait laboratory, each subject walked at a self-selected pace on an 8-m walkway and crossed a height-adjustable obstacle (Chen et al., 2004; Lu et al., 2007). The subjects were allowed to familiarize themselves with the walkway before experimental data were recorded. Twenty-eight markers were placed on the pelvis (ASISs and PSISs), as well as on each thigh (greater trochanter, mid-thigh, medial and lateral epicondyles), shank (head of fibula, tibial tuberosity, medial and lateral malleoli), and foot (navicular tuberosity, fifth metatarsal base, big toe and heel), to define the segmental coordinate systems. Kinematic data were measured using a seven-camera motion analysis system (Vicon 512, Oxford Metrics Group, UK) at a sampling rate of $120 \mathrm{~Hz}$. Two forceplates $(50.8 \mathrm{~cm} \times 46.4 \mathrm{~cm}$, OR-6-7-1000, AMTI, USA) were placed adjacent to each other with the short edges and on either side of the obstacle to measure the ground reaction forces (GRF). Based on published heel-to-heel step length data without forceplates present (Chen et al., 1991), this configuration was not expected to impose a behavioral constraint on the crossing stride. Test conditions included crossing obstacles of three different heights $(10 \%, 20 \%$, and $30 \%$ of leg length) for each limb leading. The order of obstacle height was randomly selected for each subject. For all conditions, subjects were instructed to walk along the walkway and step over the obstacle when necessary. The desired lead limb was elicited by changing the starting position. Six successful trials, three for each leg as the leading limb, for each condition were recorded.

\subsection{Data analysis}

For dynamic analysis, the pelvis-leg apparatus was modeled as a sevenlink system, each link (limb segment) embedded with an orthogonal coordinate system with the positive $x$-axis directed anteriorly, the positive $y$-axis superiorly, and the positive $z$-axis to the right. The pose of each segment was determined in terms of its transformation matrix by minimizing marker array deformation from its reference shape in a least-squares sense (Veldpaus et al., 1988). A cardanic rotation sequence $(Z-X-Y)$ was used to describe the rotational movements of each joint (Cole et al., 1993). With the measured GRF and kinematic data, intersegmental forces and internal moments at the joints of the lower limbs were calculated using inverse dynamics. Inertial properties for each body segment, namely mass, center of mass, and moment of inertia were obtained using Dempster's coefficients (Dempster et al., 1959).

Angular motions of the pelvis and the joints of both limbs, as well as joint moments of the leading limb were calculated and their values when the trailing toe was above the obstacle were extracted, namely the crossing angles of both limbs and pelvis and the crossing moments of the leading stance limb. The leading toe-out angles at the same instance were also obtained. Peak moments of the leading stance joints were calculated to indicate the maximum muscular effort at the joints.

For all the calculated variables, a mixed analysis of variance (ANOVA) with one between-subject factor (OA and control groups) and one withinsubject factor (obstacle height) was used. If a height effect was found, a polynomial test was performed to determine the trend (linear or quadratic). All significance levels were set at $\alpha=0.05$. SPSS version 11.0 (SPSS Inc., Chicago, IL, USA) was used for all statistical analyses.

\section{Results}

Demographic data of the subject groups are summarized in Table 1 . They were statistically the same except that the OA group was slightly heavier than the control group. Pain levels between limbs in the OA group were not different. 
Table 1

Characteristics of patients with bilateral medial compartment knee osteoarthritis (OA) and matched control subjects

\begin{tabular}{|c|c|c|c|c|}
\hline Variable & Patients & Control subjects & $\begin{array}{l}\text { Between-limb } \\
\text { comparisons ( } p \text {-value) }\end{array}$ & $\begin{array}{l}\text { Between-group } \\
\text { comparisons ( } p \text {-value) }\end{array}$ \\
\hline Sex (no. of females/no. of males) & $7 / 8$ & $7 / 8$ & - & \\
\hline Age, years (mean \pm S.D.) & $70.8 \pm 4.9$ & $72.0 \pm 6.0$ & - & 0.51 \\
\hline Height, $\mathrm{cm}(\operatorname{mean} \pm$ S.D.) & $160.8 \pm 9.7$ & $160 \pm 5.7$ & - & 0.72 \\
\hline Leg length, cm (mean \pm S.D.) & $82.8 \pm 5.6$ & $79.8 \pm 5.1$ & - & 0.13 \\
\hline Mass, kg (mean \pm S.D.) & $65.9 \pm 9.5$ & $58 \pm 10.4$ & - & $0.04^{*}$ \\
\hline \multicolumn{5}{|l|}{$\mathrm{K} / \mathrm{L}$ grade } \\
\hline$(3,3)$ & 11 & - & - & - \\
\hline$(3,2)$ & 3 & - & - & - \\
\hline$(4,3)$ & 1 & - & - & - \\
\hline \multicolumn{5}{|l|}{ Pain, cm $(0-10)$} \\
\hline L (mean \pm S.D. $)$ & $3.04 \pm 1.09$ & - & 0.85 & - \\
\hline $\mathrm{R}(\mathrm{mean} \pm \mathrm{S} . \mathrm{D})$. & $3.00 \pm 1.20$ & - & & - \\
\hline
\end{tabular}

$\mathrm{L}$ and $\mathrm{R}$ indicate left and right knees.

* Significant group effect using an independent $t$-test $(p<0.05)$

Table 2

Means (S.D.) of the trailing toe clearances and crossing speeds for both groups when the trailing limb crossed obstacles of three different heights

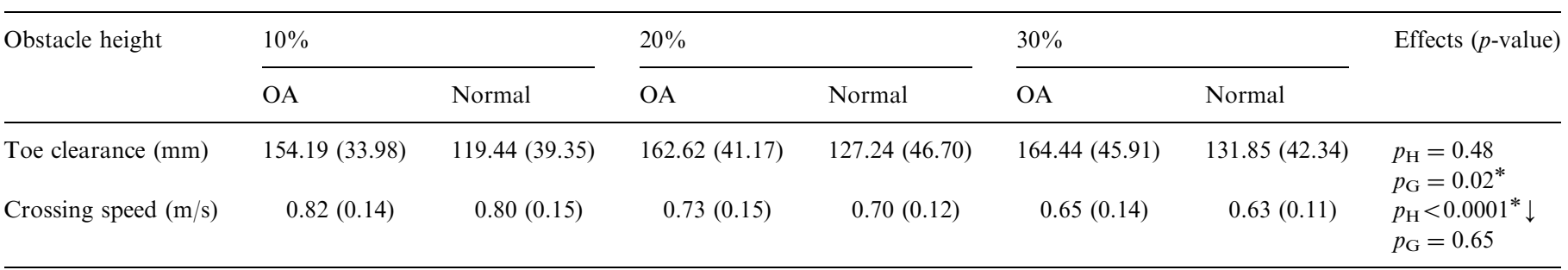

With increasing obstacle height, a downward arrow indicates a statistically significant linearly decreasing trend.

*Significant height $(\mathrm{H})$ or group $(\mathrm{G})$ effects $(p<0.05)$.

Comparisons between the OA and normal groups did not reveal any significant difference in the crossing speed (Table 2). The trailing toe clearance in the OA group was significantly higher than that in the healthy controls $(F(2,28)=6.19, p=0.022$; Table 2$)$. When the trailing toe was above the obstacle, greater hip abduction $(F(2,28)=18.74, p<0.001)$ in the trailing swing limb was found in the OA group (Table 3). Obstacle height effects were found in the swing joint angles, except for the transverse components at the hip and knee, as well as ankle eversion in both groups (Table 3$)$. For the leading stance limb, smaller hip adduction $(F(2,28)=14.41, p=0.001)$, knee flexion $(F(2,28)=5.75, p=0.024)$, and ankle eversion $(F(2,28)=4.57, p=0.04)$ were found in the OA group (Table 4). Joint angles of the leading stance limb were also affected by obstacle height for the hip adduction $(F(2,28)=74.41, \quad p<0.001) \quad$ and external rotation $(F(2,28)=36.12, \quad p<0.001), \quad$ as well as knee flexion $(F(2,28)=32.53, \quad p<0.001) \quad$ and $\quad$ knee abduction $(F(2,28)=32.53, p<0.001)$ for both groups (Table 4). Greater pelvic anterior tilt $(F(2,28)=4.46, \quad p=0.04)$ and toe-out angles $(F(2,28)=4.79, \quad p=0.04)$ were also found in the OA group (Table 5). When the obstacle height increased, both groups linearly increased their pelvic anterior tilt $(F(2,28)=63.81, p<0.001)$, swing-side backward rotation $(F(2,28)=32.92, p<0.001)$, and list angles $(F(2,28)=64.22, p<0.001)$.

Peak and crossing moments of the ankle joint were statistically the same for both groups. During early stance, greater knee abductor moments were found in the OA group $(F(2,28)=6.32, p=0.02$; Fig. 1$)$. When the trailing toe was above the obstacle, greater knee abductor crossing moments were also found in the OA group $(F(2,28)=4.34$, $p=0.04)$. During late stance, greater peak hip abductor moments were also found in the OA group $(F(2,28)=5.46$, $p=0.03$ ). Height effects were found in early peak and crossing hip abductor moments (early stance: $F(2,28)=35.74, \quad p<0.001 ; \quad$ crossing: $\quad F(2,28)=22.26$, $p<0.001)$, as well as in late peak knee abductor moments $(F(2,28)=11.98, p=0.002)$ for both groups.

Ankle moments in the sagittal plane were not significantly different between groups. Greater hip extensor moments (early stance: $F(2,28)=4.27, p=0.04$; crossing: $F(2,28)=11.98, p=0.002$; late stance: $F(2,28)=11.61$, $p=0.002)$ and smaller knee extensor moments (early stance: $F(2,28)=22.57, \quad p<0.001 ; \quad$ crossing: $F(2,28)=23.13, p<0.0001$; late stance: $F(2,28)=19.51$, $p<0.001$ ) were found in the OA group (Fig. 2). Height effects were found in the peak hip extensor moments during early stance $(F(2,28)=35.74, p<0.001)$ and hip 
Table 3

Crossing angles of the joints of the trailing swing limb when the trailing toe was above the obstacle

\begin{tabular}{|c|c|c|c|c|c|c|c|c|}
\hline \multirow{3}{*}{$\begin{array}{l}\text { Crossing angle } \\
\text { (deg) }\end{array}$} & \multirow[t]{3}{*}{ Group } & \multicolumn{6}{|c|}{ Obstacle height } & \multirow{3}{*}{$\begin{array}{l}\text { Effects (height and } \\
\text { group) ( } p \text {-value) }\end{array}$} \\
\hline & & \multicolumn{2}{|l|}{$10 \%$} & \multicolumn{2}{|l|}{$20 \%$} & \multicolumn{2}{|l|}{$30 \%$} & \\
\hline & & Mean & S.D. & Mean & S.D. & Mean & S.D. & \\
\hline \multicolumn{9}{|l|}{ Hip } \\
\hline \multirow[t]{2}{*}{ Flex } & $\mathrm{OA}$ & 25.47 & 3.58 & 29.34 & 6.31 & 32.48 & 8.68 & $p_{\mathrm{H}}<0.001^{*} \uparrow$ \\
\hline & Normal & 24.48 & 6.03 & 29.98 & 5.73 & 33.92 & 6.01 & $p_{\mathrm{G}}=0.86$ \\
\hline \multirow[t]{2}{*}{ Add } & $\mathrm{OA}$ & -2.47 & 3.95 & -2.4 & 3.80 & -4.31 & 3.83 & $p_{\mathrm{H}}<0.001^{*} \uparrow$ \\
\hline & Normal & 3.47 & 3.81 & 4.43 & 5.74 & 4.03 & 6.88 & $p_{\mathrm{G}}=0.02^{*}$ \\
\hline \multirow[t]{2}{*}{ IR } & OA & 2.04 & 4.32 & 3.89 & 4.99 & 5.52 & 5.57 & $p_{\mathrm{H}}=0.18$ \\
\hline & Normal & 2.85 & 4.94 & 2.43 & 5.62 & 2.39 & 6.40 & $p_{\mathrm{G}}=0.47$ \\
\hline \multicolumn{9}{|l|}{ Knee } \\
\hline \multirow[t]{2}{*}{ Flex } & $\mathrm{OA}$ & 96.24 & 8.11 & 109.61 & 9.51 & 118.83 & 8.52 & $p_{\mathrm{H}}<0.001^{*} \uparrow$ \\
\hline & Normal & 95.95 & 8.77 & 112.51 & 8.96 & 124.6 & 7.63 & $p_{\mathrm{G}}=0.29$ \\
\hline \multirow[t]{2}{*}{ Abd } & $\mathrm{OA}$ & 6.11 & 4.54 & 4.55 & 3.97 & 4.15 & 4.10 & $p_{\mathrm{H}}=0.003^{*} \downarrow$ \\
\hline & Normal & 15.11 & 9.67 & 13.60 & 10.34 & 12.40 & 9.27 & $p_{\mathrm{G}}=0.053$ \\
\hline \multirow[t]{2}{*}{ ER } & $\mathrm{OA}$ & 15.10 & 6.76 & 15.26 & 7.97 & 16.13 & 9.22 & $p_{\mathrm{H}}=0.19$ \\
\hline & Normal & 21.50 & 6.67 & 22.72 & 8.84 & 23.37 & 10.49 & $p_{\mathrm{G}}=0.23$ \\
\hline \multicolumn{9}{|l|}{ Ankle } \\
\hline \multirow[t]{2}{*}{ Dorsi } & $\mathrm{OA}$ & 1.91 & 8.29 & 4.84 & 7.18 & 7.78 & 6.96 & $p_{\mathrm{H}}<0.001^{*} \uparrow$ \\
\hline & Normal & 2.46 & 7.47 & 7.02 & 9.02 & 10.79 & 7.83 & $p_{\mathrm{G}}=0.49$ \\
\hline \multirow[t]{2}{*}{ Eve } & $\mathrm{OA}$ & -0.2 & 4.57 & 0.13 & 4.92 & 0.09 & 5.64 & $p_{\mathrm{H}}=0.6$ \\
\hline & Normal & 2.01 & 2.79 & 2.32 & 3.59 & 2.51 & 3.29 & $p_{\mathrm{G}}=0.13$ \\
\hline \multirow[t]{2}{*}{ IR } & $\mathrm{OA}$ & -0.81 & 6.21 & 2.56 & 6.25 & 6.33 & 7.33 & $p_{\mathrm{H}}=0.09$ \\
\hline & Normal & 0.37 & 3.19 & -0.82 & 5.67 & 0.43 & 4.23 & $p_{\mathrm{G}}=0.15$ \\
\hline
\end{tabular}

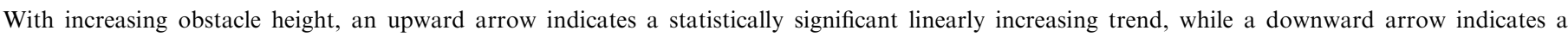

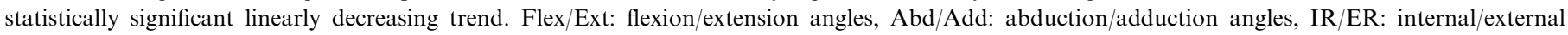
angles.

${ }^{*}$ Significant height $(\mathrm{H})$ or group $(\mathrm{G})$ effects $(p<0.05)$.

crossing extensor moments $(F(2,28)=22.26, p<0.001)$, as well as in peak knee moments during early and late stance (early stance: $F(2,28)=7.73, p=0.003$; late stance: $F(2,28)=15.84, \quad p<0.001)$ and in the knee crossing flexor moments $(F(2,28)=49.52, p<0.001)$ for both groups (Fig. 2).

\section{Discussion}

The current study aimed to investigate the biomechanical strategies adopted by older adults with medial compartment knee OA for successful obstacle crossing by the trailing limb. The OA group successfully crossed the obstacle with higher trailing toe clearance (Table 2). This may be helpful for reducing the probability of the foot hitting the obstacle and thus decreasing the risk of tripping, when neither the swing limb nor the obstacle is within the subject's visual field. Particular kinematic and kinetic changes in the pelvis-leg apparatus were needed for this altered end-point control.

Apart from the increased anterior pelvic tilt, changes in joint kinematics also contributed directly to the greater trailing toe clearance in the OA group, namely decreased knee flexion and hip adduction of the leading stance limb, and increased hip abduction of the swing limb. These changes were also found among other kinematic changes of the joints in both groups (Tables 3 and 4) to ensure a constant toe clearance when facing obstacles of increasing height. This suggests that these changes were the primary strategies that the OA subjects used when it was necessary to increase the foot height during swing. These strategies emerged possibly as a result of interactions between factors such as volition, neurological control, musculoskeletal conditions, and pain to meet the mechanical and safety demands. When the swing toe was above the obstacle, the swing hip was in an abducted position in the OA group, while in an adducted position in the normal group. Since the varus alignment of the OA knee may reduce the distance between the swing toe and the stance limb, abduction of the swing hip in the OA group seemed to be helpful for reducing the probability of the swing toe hitting the stance limb when the trailing limb was crossing. Although swing ankle eversion was also a possible strategy to increase the distance between the swing toe and the 
Table 4

Crossing angles of the joints of the leading stance limb when the trailing toe was above the obstacle

\begin{tabular}{|c|c|c|c|c|c|c|c|c|}
\hline \multirow{3}{*}{$\begin{array}{l}\text { Crossing angle } \\
\text { (deg) }\end{array}$} & \multirow[t]{3}{*}{ Group } & \multicolumn{6}{|c|}{ Obstacle height } & \multirow{3}{*}{$\begin{array}{l}\text { Effects (height and } \\
\text { group) ( } p \text {-value) }\end{array}$} \\
\hline & & \multicolumn{2}{|l|}{$10 \%$} & \multicolumn{2}{|l|}{$20 \%$} & \multicolumn{2}{|l|}{$30 \%$} & \\
\hline & & Mean & S.D. & Mean & S.D. & Mean & S.D. & \\
\hline \multicolumn{9}{|l|}{ Hip } \\
\hline \multirow[t]{2}{*}{ Flex } & $\mathrm{OA}$ & 10.71 & 4.20 & 10.02 & 4.40 & 10.08 & 4.87 & $p_{\mathrm{H}}=0.14$ \\
\hline & Normal & 11.41 & 4.08 & 10.35 & 4.01 & 10.94 & 4.64 & $p_{\mathrm{G}}=0.68$ \\
\hline \multirow[t]{2}{*}{ Add } & $\mathrm{OA}$ & 5.14 & 2.39 & 2.78 & 3.41 & -0.77 & 4.64 & $p_{\mathrm{H}}<0.001^{*} \downarrow$ \\
\hline & Normal & 10.69 & 4.03 & 8.15 & 4.23 & 3.99 & 5.34 & $p_{\mathrm{G}}=0.001^{*}$ \\
\hline \multirow{2}{*}{ ER } & $\mathrm{OA}$ & 0.42 & 5.59 & 1.03 & 5.27 & 3.79 & 6.01 & $p_{\mathrm{H}}<0.001^{*} \uparrow$ \\
\hline & Normal & -0.61 & 4.24 & 0.74 & 3.75 & 3.21 & 3.98 & $p_{\mathrm{G}}=0.71$ \\
\hline \multicolumn{9}{|l|}{ Кnеe } \\
\hline \multirow[t]{2}{*}{ Flex } & $\mathrm{OA}$ & 5.84 & 4.60 & 4.34 & 5.28 & 2.80 & 4.36 & $p_{\mathrm{H}}=<0.001^{*} \downarrow$ \\
\hline & Normal & 10.43 & 5.27 & 7.80 & 4.24 & 6.77 & 3.66 & $p_{\mathrm{G}}=0.02^{*}$ \\
\hline \multirow[t]{2}{*}{ Abd } & $\mathrm{OA}$ & 0.74 & 2.54 & 0.75 & 2.64 & 0.57 & 2.68 & $p_{\mathrm{H}}=0.02^{*} \downarrow$ \\
\hline & Normal & 2.61 & 3.23 & 1.99 & 2.84 & 1.57 & 2.98 & $p_{\mathrm{G}}=0.21$ \\
\hline \multirow[t]{2}{*}{ ER } & $\mathrm{OA}$ & 0.37 & 1.59 & 0.17 & 1.87 & -0.33 & 2.03 & $p_{\mathrm{H}}=0.07$ \\
\hline & Normal & -0.14 & 4.58 & -1.04 & 4.64 & -1.17 & 5.39 & $p_{\mathrm{G}}=0.51$ \\
\hline \multicolumn{9}{|l|}{ Ankle } \\
\hline \multirow[t]{2}{*}{ Dorsi } & $\mathrm{OA}$ & 2.87 & 4.07 & 3.02 & 3.58 & 3.26 & 3.55 & $p_{\mathrm{H}}=0.59$ \\
\hline & Normal & 4.00 & 2.96 & 3.79 & 3.27 & 2.93 & 4.45 & $p_{\mathrm{G}}=0.69$ \\
\hline \multirow[t]{2}{*}{ Eve } & $\mathrm{OA}$ & 2.93 & 1.99 & 2.83 & 2.66 & 2.04 & 2.76 & $p_{\mathrm{H}}=0.38$ \\
\hline & Normal & 4.5 & 2.2 & 4.25 & 2.98 & 4.29 & 2.77 & $p_{\mathrm{G}}=0.04^{*}$ \\
\hline \multirow[t]{2}{*}{ IR } & $\mathrm{OA}$ & 1.37 & 2.36 & 1.5 & 3.13 & 0.45 & 3.07 & $p_{\mathrm{H}}=0.19$ \\
\hline & Normal & 0.14 & 2.42 & 0.82 & 2.84 & 0.59 & 3.14 & $p_{\mathrm{G}}=0.55$ \\
\hline
\end{tabular}

With increasing obstacle height, an upward arrow indicates a statistically significant linearly increasing trend, while a downward arrow indicates a statistically significant linearly decreasing trend. Flex/Ext: flexion/extension angles, Abd/Add: abduction/adduction angles, IR/ER: internal/external angles.

${ }^{*}$ Significant height $(\mathrm{H})$ or group $(\mathrm{G})$ effects $(p<0.05)$.

Table 5

Pelvic and toe-out angles when the trailing toe was above the obstacle

\begin{tabular}{|c|c|c|c|c|c|c|c|c|}
\hline \multirow[t]{3}{*}{ Angle (deg) } & \multirow[t]{3}{*}{ Group } & \multicolumn{6}{|c|}{ Obstacle height } & \multirow{3}{*}{$\begin{array}{l}\text { Effects (height and } \\
\text { group) ( } p \text {-value) }\end{array}$} \\
\hline & & \multicolumn{2}{|l|}{$10 \%$} & \multicolumn{2}{|l|}{$20 \%$} & \multicolumn{2}{|l|}{$30 \%$} & \\
\hline & & Mean & S.D. & Mean & S.D. & Mean & S.D. & \\
\hline \multicolumn{9}{|l|}{ Pelvic } \\
\hline Anterior tilt & $\begin{array}{l}\text { OA } \\
\text { Normal }\end{array}$ & $\begin{array}{l}16.10 \\
11.70\end{array}$ & $\begin{array}{l}4.85 \\
3.88\end{array}$ & $\begin{array}{l}17.18 \\
13.47\end{array}$ & $\begin{array}{l}4.60 \\
4.50\end{array}$ & $\begin{array}{l}18.93 \\
15.49\end{array}$ & $\begin{array}{l}5.09 \\
4.21\end{array}$ & $\begin{array}{l}p_{\mathrm{H}}<0.001^{*} \uparrow \\
p_{\mathrm{G}}=0.04^{*}\end{array}$ \\
\hline $\begin{array}{l}\text { Swing-side } \\
\text { backward } \\
\text { rotation }\end{array}$ & $\begin{array}{l}\text { OA } \\
\text { Normal }\end{array}$ & $\begin{array}{l}4.89 \\
5.82\end{array}$ & $\begin{array}{l}2.88 \\
2.52\end{array}$ & $\begin{array}{l}7.08 \\
7.84\end{array}$ & $\begin{array}{l}3.69 \\
3.01\end{array}$ & $\begin{array}{l}11.78 \\
12.92\end{array}$ & $\begin{array}{l}8.16 \\
6.61\end{array}$ & $\begin{array}{l}p_{\mathrm{H}}<0.001^{*} \uparrow \\
p_{\mathrm{G}}=0.6\end{array}$ \\
\hline Swing-side list & $\begin{array}{l}\text { OA } \\
\text { Normal }\end{array}$ & $\begin{array}{l}1.24 \\
0.05\end{array}$ & $\begin{array}{l}2.04 \\
1.84\end{array}$ & $\begin{array}{l}3.77 \\
2.92\end{array}$ & $\begin{array}{l}3.29 \\
2.82\end{array}$ & $\begin{array}{l}7.69 \\
6.94\end{array}$ & $\begin{array}{l}5.50 \\
4.22\end{array}$ & $\begin{array}{l}p_{\mathrm{H}}<0.001^{*} \uparrow \\
p_{\mathrm{G}}=0.4\end{array}$ \\
\hline Toe-out & $\begin{array}{l}\text { OA } \\
\text { Normal }\end{array}$ & $\begin{array}{l}9.93 \\
7.07\end{array}$ & $\begin{array}{l}6.57 \\
3.88\end{array}$ & $\begin{array}{r}11.50 \\
5.59\end{array}$ & $\begin{array}{l}5.71 \\
3.49\end{array}$ & $\begin{array}{r}11.57 \\
7.46\end{array}$ & $\begin{array}{l}6.01 \\
3.54\end{array}$ & $\begin{array}{l}p_{\mathrm{H}}=0.43 \\
p_{\mathrm{G}}=0.04^{*}\end{array}$ \\
\hline
\end{tabular}

With increasing obstacle height, an upward arrow indicates a statistically significant linearly increasing trend, while a downward arrow indicates a statistically significant linearly decreasing trend.

*Significant height $(\mathrm{H})$ or group $(\mathrm{G})$ effects $(p<0.05)$. 

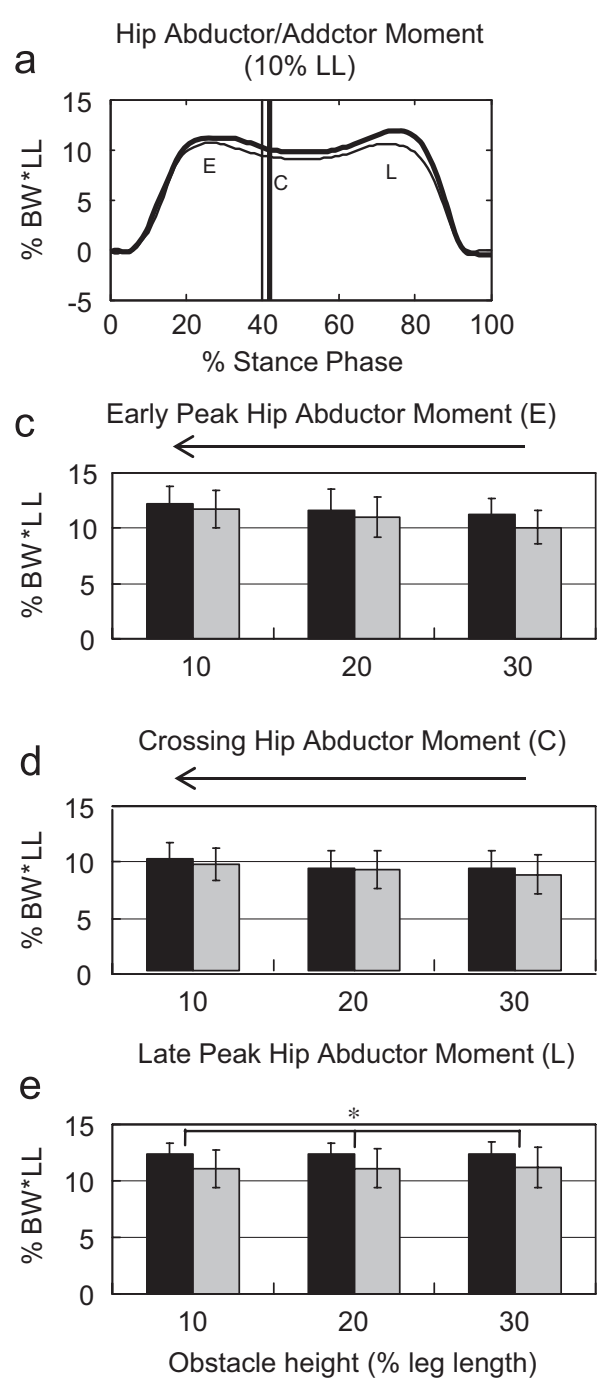

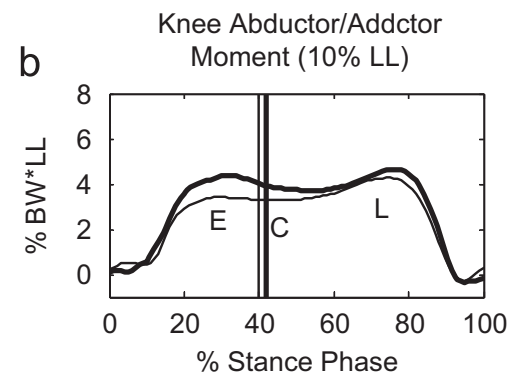

f Early Peak Knee Abductor Moment (E)

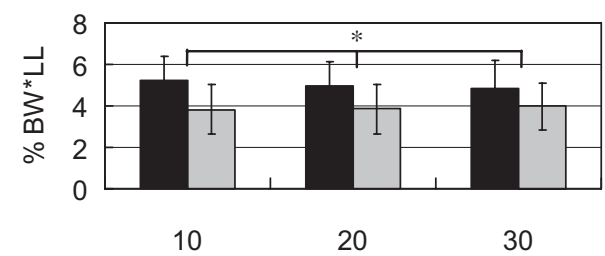

g

Crossing Knee Abductor Moment (C)
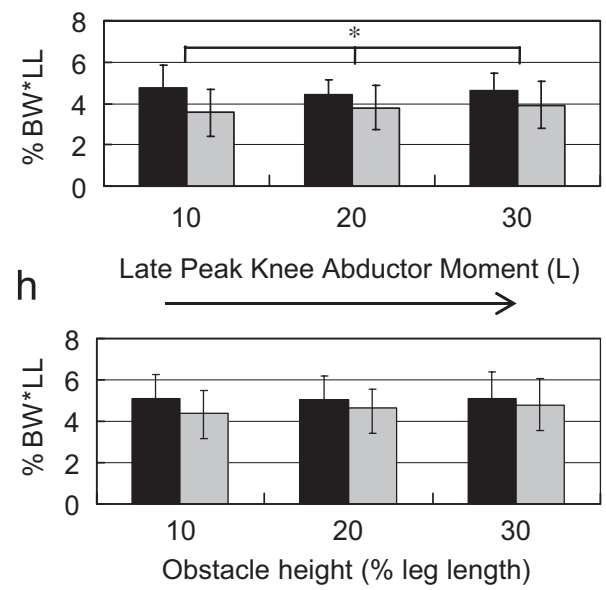

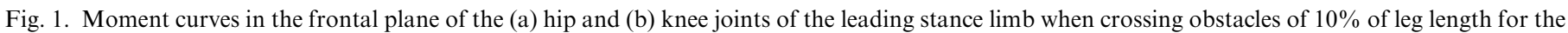

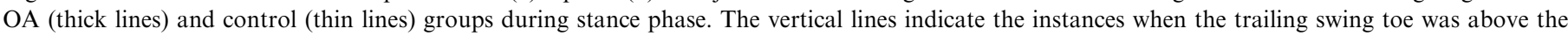

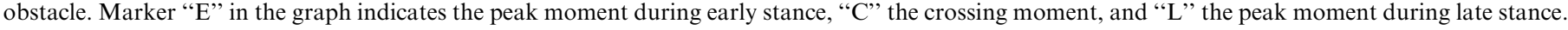

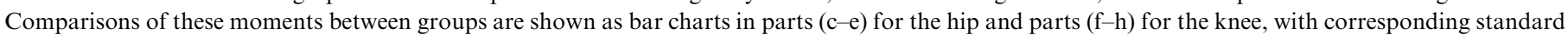

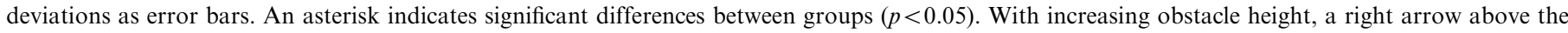

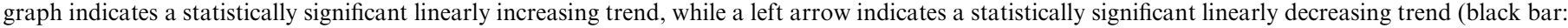
the OA group, gray bar: the control group).

stance limb, abduction of the swing hip was more effective because the hip is much more proximal than the ankle.

Greater knee abductor moments were needed in the OA group before and when the swing toe passed over the obstacle, and returned to a normal level afterwards. Greater knee abductor moments may be associated with varus alignment of the diseased knee, which would shift the line of action of the GRF medial to the knee joint center. During late stance, the knee abductor moments in the OA group were similar to those in the healthy controls, most likely due to the increased toe-out angle. A greater toe-out angle will result in orienting the GRF to pass closer to the knee joint center, thus decreasing the second peak knee abductor moment during late stance (Hurwitz et al., 2002). Increased peak hip abductor moments during late stance may be associated with the reduced peak knee abductor moments. Strategies for reducing knee abductor moments in the OA group may be helpful for reducing joint loading and associated pain at the knee. Pain relief was reported to decrease the risk of tripping over obstacles (Pandya et al., 2007). Potential remedies may include knee bracing and corrective footwear that were reported to be useful for decreasing knee abductor moments during level walking (Pollo et al., 2002; Toda and Tsukimura, 2004).

Changes in the knee abductor moments in the current study were similar to those during leading limb crossing (Lu et al., 2007), except that the knee abductor crossing moment was greater than normal when the trailing toe was above the obstacle. During leading limb crossing, increased stance hip abduction and swing-side pelvic list were 

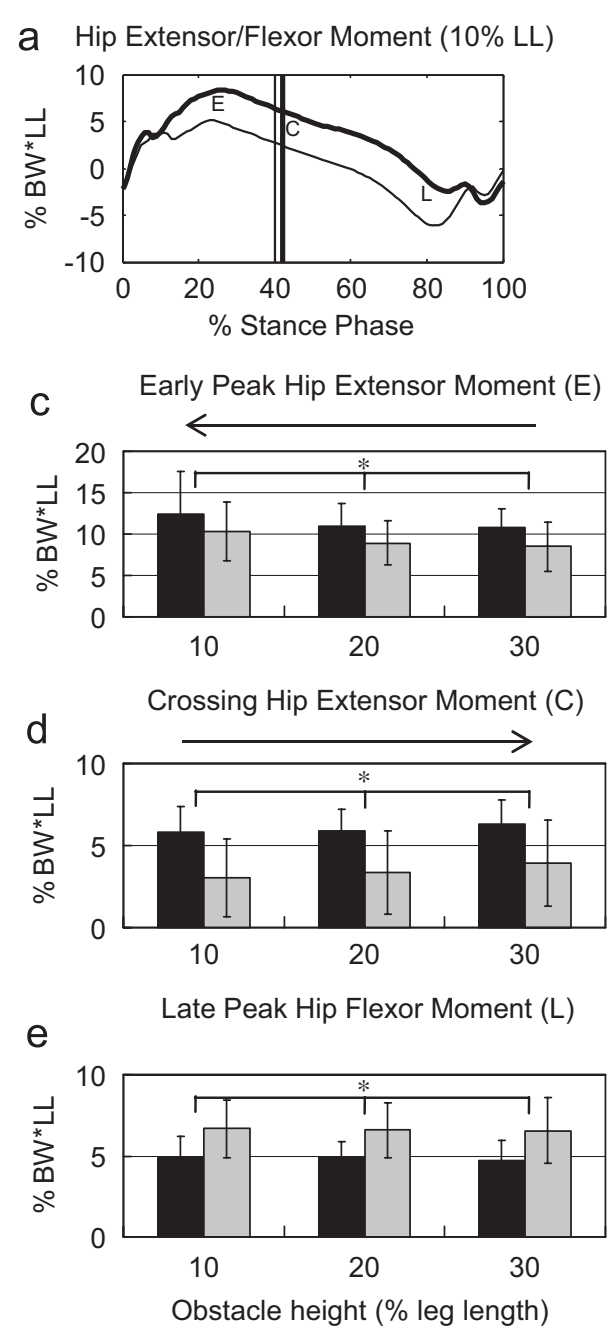
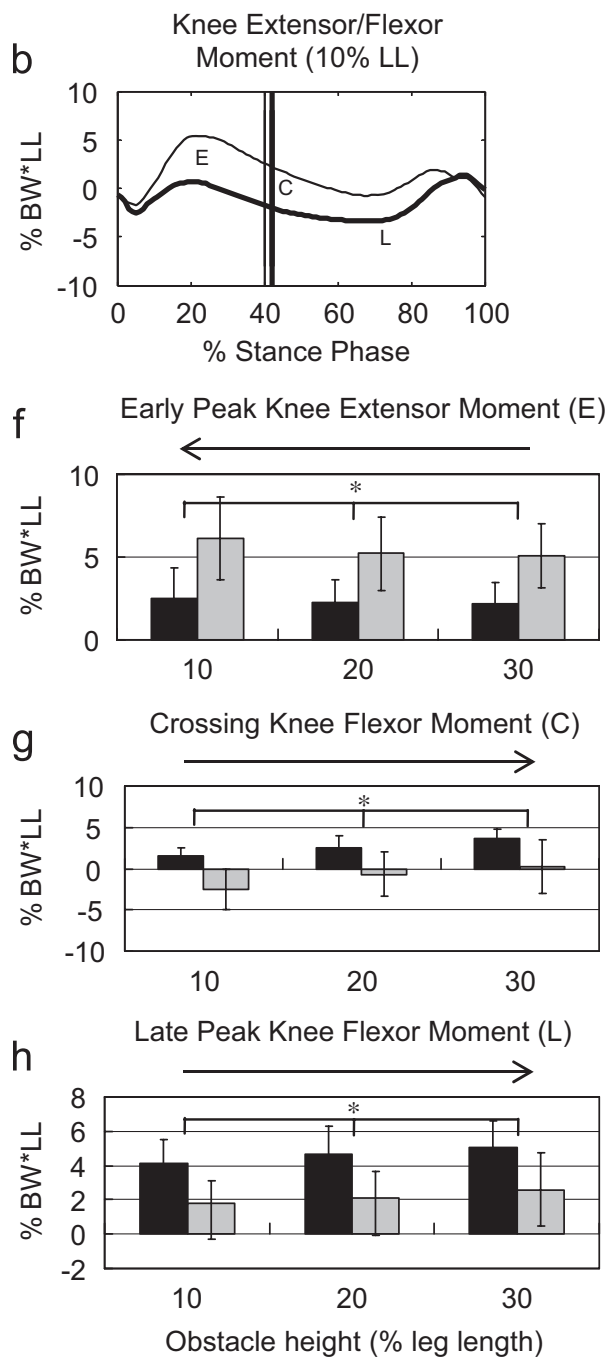

Fig. 2. Moment curves in the sagittal plane of the (a) hip and (b) knee joints of the leading stance limb when crossing obstacles of $10 \%$ of leg length for the $\mathrm{OA}$ (thick lines) and control (thin lines) groups during stance phase. The vertical lines indicate the instances when the trailing swing toe was above the obstacle. Marker " $E$ " in the graph indicates the peak moment during early stance, " $C$ " the crossing moment, and "L" the peak moment during late stance. Comparisons of these moments between groups are shown as bar charts in parts (c-e) for the hip and parts (f-h) for the knee, with corresponding standard deviations as error bars. An asterisk indicates significant differences between groups $(p<0.05)$. With increasing obstacle height, a right arrow above the graph indicates a statistically significant linearly increasing trend, while a left arrow indicates a statistically significant linearly decreasing trend (black bar: the OA group, gray bar: the control group).

adopted in the OA group to shift the GRF line closer to the stance knee center ( $\mathrm{Lu}$ et al., 2007), resulting in normal knee joint loads. However, this compensation was not present when the trailing limb was crossing. This may be due to the quite different postural, and thus mechanical, demands between the two phases of obstacle crossing. The swing limb is mainly ahead of the stance limb during leading limb crossing with the COM moving away from the stance limb, while the swing limb follows the stance limb during trailing limb crossing with the COM moving towards the supporting limb (Chen and Lu, 2006). Apart from differences in mechanical demands, other factors such as impaired proprioception, pain, and limitation of joint motion could also have contributed to the observed differences between leading versus trailing limb obstacle crossing. However, since the subjects had bilateral OA with similar severity and pain levels, the effects of these associated impairments may be limited.

The OA group also exhibited different joint kinetics in the sagittal plane compared to the normal group. Smaller knee extensor moments were used in the OA group throughout the stance phase, which may be due to the weakness of the quadriceps accompanying knee OA (O'Reilly et al., 1998). When the trailing toe was above the obstacle, greater knee flexor crossing moments (possibly as a result of the weakened knee extensors) were observed in the OA group, compared to knee extensor crossing moments in the healthy control. In order to compensate for the physical condition of the OA knee, including muscle weakness and malalignment, the OA group appeared to flex the stance knee less and tilted the pelvis more anteriorly to control the stability of the stance 
knee. A more extended knee position also contributed to the higher trailing toe clearance in the OA group. The OA group also increased the hip extensor effort to ensure body stability under the altered posture and to prevent the body from experiencing too much forward progression owing to the instability of the OA knee. The same strategies were also found during leading limb crossing ( $\mathrm{Lu}$ et al., 2007). This is in contrast to the joint kinetics in the frontal plane where a different kinetic strategy between the leading and trailing limbs was needed. To help reinforce the performance of the leading stance limb for successful obstacle crossing, the use of a knee brace may be considered because knee bracing has been shown to increase the proprioception and stability of the OA knee (Birmingham et al., 2001).

In the current study, the effects from selection bias were reduced by adopting homogeneous subjects and matching between groups, except for the slightly higher body weight in the OA group, reflecting the fact that overweight is a risk factor for knee OA (Felson et al., 1988). However, this approach inevitably limits the generalization of the current findings. For example, patients with excessive pain and/or with different pain between the two limbs may have to develop different strategies for successful crossing. For patients with unilateral and/or lateral compartment knee $\mathrm{OA}$, the biomechanical strategies adopted may be different too. Gender, severity of OA, and walking speed may also be factors. Further study is needed to reveal the potential differences between these groups and the effects of the possible factors.

In conclusion, higher trailing toe clearance was adopted by the OA group, which may be helpful for decreasing the risk of tripping when neither the swing limb nor the obstacle is within the subject's visual field when the trailing toe is crossing. In order to achieve this higher toe clearance, particular joint kinematic and kinetic strategies have been adopted in the OA group. Weakness in the hip abductors and extensors in individuals with OA may be risk factors for tripping owing to the greater demands on these muscle groups during obstacle crossing by these individuals.

\section{Conflict of interest statement}

We, Hao-Ling Chen, Tung-Wu Lu, Ting-Ming Wang and Shier-Chieg Huang, declare that we have no proprietary, financial, professional or other personal interest of any nature or kind in any product, service and/or company that could be construed as influencing the position presented in, or the review of, the manuscript entitled, "Biomechanical strategies for successful obstacle crossing with the trailing limb in older adults with medial compartment knee osteoarthritis."

\section{Acknowledgments}

The authors gratefully acknowledge the support from the National Science Council (NSC95-2314-B002-
167-MY2) and National Health Research Institutes (NHRI-EX91-9126EP) of Taiwan.

\section{References}

Birmingham, T.B., Kramer, J.F., Kirkley, A., Inglis, J.T., Spaulding, S.J., Vandervoort, A.A., 2001. Knee bracing for medial compartment osteoarthritis: effects on proprioception and postural control. Rheumatology 40, 285-289.

Blake, A.J., Morgan, K., Bendall, M.J., Dallosso, H., Ebrahim, S.B., Arie, T.H., Fentem, P.H., Bassey, E.J., 1988. Falls by elderly people at home: prevalence and associated factors. Age and Ageing 17, 365-372.

Campbell, A.J., Borrie, M.J., Spears, G.F., Jackson, S.L., Brown, J.S., Fitzgerald, J.L., 1990. Circumstances and consequences of falls experienced by a community population 70 years and over during a prospective study. Age and Ageing 19, 136-141.

Chen, H.L., Lu, T.W., 2006. Comparisons of the joint moments between leading and trailing limb in young adults when stepping over obstacles. Gait and Posture 23, 69-77.

Chen, H.C., Ashton-Miller, J.A., Alexander, N.B., Schultz, A.B., 1991 Stepping over obstacles: gait patterns of healthy young and old adults. Journals of Gerontology Series A-Biological Sciences and Medical Sciences 46, M196-M203.

Chen, H.L., Lu, T.W., Lin, H.C., 2004. Three-dimensional kinematic analysis of stepping over obstacles in young subjects. Biomedical Engineering-Applications, Basis and Communications 16, 157-164.

Chou, L.S., Draganich, L.F., 1997. Stepping over an obstacle increases the motions and moments of the joints of the trailing limb in young adults. Journal of Biomechanics 30, 331-337.

Cole, G.K., Nigg, B.M., Ronsky, J.L., Yeadon, M.R., 1993. Application of the joint coordinate system to three-dimensional joint attitude and movement representation: a standardization proposal. Journal of Biomechanical Engineering 115, 344-349.

Dalton, J.A., McNaull, F., Dalton, J.A., McNaull, F., 1998. A call for standardizing the clinical rating of pain intensity using a $0-10$ rating scale. Cancer Nursing 21, 46-49.

Dempster, W.T., Gabel, W.C., Felts, W.J.L., 1959. The anthropometry of manual work space for the seated subjects. American Journal of Physical Anthropology 17, 289-317.

Draganich, L.F., Kuo, C.E., 2004. The effects of walking speed on obstacle crossing in healthy young and healthy older adults. Journal of Biomechanics 37, 889-896.

Felson, D.T., Anderson, J.J., Naimark, A., Walker, A.M., Meenan, R.F., Felson, D.T., Anderson, J.J., Naimark, A., Walker, A.M., Meenan, R.F., 1988. Obesity and knee osteoarthritis. The Framingham study. Annals of Internal Medicine 109, 18-24.

Hoyert, D.L., Arias, E., Smith, B.L., Murphy, S.L., Kochanek, K.D., 2001. Deaths: final data for 1999. National Vital Statistics Reports, vol. 49, pp. 1-113.

Hurwitz, D.E., Ryals, A.B., Case, J.P., Block, J.A., Andriacchi, T.P., 2002. The knee adduction moment during gait in subjects with knee osteoarthritis is more closely correlated with static alignment than radiographic disease severity, toe out angle and pain. Journal of Orthopaedic Research 20, 101-107.

Kellgren, J.H., Lawrence, J.S., 1957. Radiological assessment of osteoarthritis. Annals of the Rheumatic Diseases 16, 494-502.

Leveille, S.G., Bean, J., Bandeen-Roche, K., 2002. Musculoskeletal pain and risk for falls in older disabled woman living in the community. Journal of the American Geriatrics Society 50, 671-678.

Lu, T.W., Chen, H.L., Chen, S.C., 2006. Comparisons of the lower limb kinematics between young and older adults when crossing obstacles of different heights. Gait and Posture 23, 471-479.

Lu, T.W., Chen, H.L., Wang, T.M., 2007. Obstacle crossing in older adults with medial compartment knee osteoarthritis. Gait and Posture 26, 553-559.

McFadyen, B.J., Prince, F., 2002. Avoidance and accommodation of surface height changes by healthy, community-dwelling, young, and 
elderly men. Journals of Gerontology Series A-Biological Sciences and Medical Sciences 57, B166-B174.

O'Reilly, S.C., Jones, A., Muir, K.R., Doherty, M., 1998. Quadriceps weakness in knee osteoarthritis: the effect on pain and disability. Annals of the Rheumatic Diseases 57, 588-594.

Overstall, P.W., Exton-Smith, A.N., Imms, F.J., Johnson, A.L., 1977. Falls in the elderly related to postural imbalance. British Medical Journal 1, 261-264.

Pandya, N.K., Draganich, L.F., Mauer, A., Piotrowski, G.A., Pottenger, L., Pandya, N.K., Draganich, L.F., Mauer, A., Piotrowski, G.A., Pottenger, L., 2005. Osteoarthritis of the knees increases the propensity to trip on an obstacle. Clinical Orthopaedics \& Related Research, 150-156.

Pandya, N.K., Piotrowski, G.A., Pottenger, L., Draganich, L.F., Pandya, N.K., Piotrowski, G.A., Pottenger, L., Draganich, L.F., 2007. Pain relief in knee osteoarthritis reduces the propensity to trip on an obstacle. Gait and Posture 25, 106-111.
Pollo, F.E., Otis, J.C., Backus, S.I., Warren, R.F., Wickiewicz, T.L., 2002. Reduction of medial compartment loads with valgus bracing of the osteoarthritic knee. American Journal of Sports Medicine 30, 414-421.

Robbins, A.S., Rubenstein, L.Z., Josephson, K.R., Schulman, B.L., Osterweil, D., Fine, G., 1989. Predictors of falls among elderly people. Results of two population-based studies. Archives of Internal Medicine 149, 1628-1633.

Tinetti, M.E., Speechley, M., Ginter, S.F., 1988. Risk factors for falls among elderly persons living in the community. New England Journal of Medicine 319, 1701-1707.

Toda, Y., Tsukimura, N., 2004. A six-month followup of a randomized trial comparing the efficacy of a lateral-wedge insole with subtalar strapping and an in-shoe lateral-wedge insole in patients with varus deformity osteoarthritis of the knee. Arthritis and Rheumatism 50, 3129-3136.

Veldpaus, F.E., Woltring, H.J., Dortmans, L.J., 1988. A least-squares algorithm for the equiform transformation from spatial marker co-ordinates. Journal of Biomechanics 21, 45-54. 